\title{
Heterologous expression of heat stress- responsive AtPLC9 confers heat tolerance in transgenic rice
}

\author{
Yuliang Liu ${ }^{1 \dagger}$, Xinye Liư ${ }^{1 \dagger}$, Xue Wang ${ }^{1}$, Kang Gao ${ }^{1}$, Weiwei Qi ${ }^{1}$, Huimin Ren ${ }^{1}$, Haorui Hu ${ }^{1,2}$, Daye Sun ${ }^{1}$,
} Jiaoteng Bai ${ }^{1,3^{*}}$ and Shuzhi Zheng ${ }^{1,3^{*}}$ (i)

\begin{abstract}
Background: As global warming becomes increasingly severe, it is urgent that we enhance the heat tolerance of crops. We previously reported that Arabidopsis thaliana PHOSPHOINOSITIDE-SPECIFIC PHOSPHOLIPASE C9 (AtPLC9) promotes heat tolerance.

Results: In this study, we ectopically expressed AtPLC9 in rice to examine its potential to improve heat tolerance in this important crop. Whereas AtPLC9 did not improve rice tolerance to salt, drought or cold, transgenic rice did exhibit greater heat tolerance than the wild type. High-throughput RNA-seq revealed extensive and dynamic transcriptome reprofiling in transgenic plants after heat stress. Moreover, the expression of some transcription factors and calcium ion-related genes showed specific upregulation in transgenic rice after heat stress, which might contribute to the enhanced heat tolerance.

Conclusions: This study provides preliminary guidance for using AtPLC9 to improve heat tolerance in cereal crops and, more broadly, highlights that heterologous transformation can assist with molecular breeding.
\end{abstract}

Keywords: AtPLC9, $\mathrm{Ca}^{2}{ }^{+}$, Heterologous expression, Rice, Thermotolerance

\section{Background}

Plants must cope with a complicated and varied environment. The primary abiotic stresses plants facing include heat, cold, drought and salt. Temperature affects plant growth, development, and geographical distribution, and extreme temperatures can adversely affect crop quality and productivity. As temperatures increase above the optimum, plants begin to experience heat stress (HS). Plants perceive and transmit HS signals through a complicated pathway. Heat stress initially increases the fluidity of

\footnotetext{
* Correspondence: jtbai@hebtu.edu.cn; szzheng@hebtu.edu.cn

${ }^{\dagger}$ Yuliang Liu and Xinye Liu contributed equally to this work.

${ }^{1}$ Ministry of Education Key Laboratory of Molecular and Cellular Biology,

Hebei Collaboration Innovation Center for Cell Signaling, Hebei Key

Laboratory of Molecular and Cellular Biology, College of Life Sciences, Hebei Normal University, Shijiazhuang 050024, China

Full list of author information is available at the end of the article
}

the plasma membrane, and researchers have proposed that HS activates cyclic nucleotide-gated channel family proteins through these changes in membrane fluidity $[1,2]$. While it is still not clear what the HS signal transduction comprises, a number of HS signal components capable of regulating thermotolerance in plants have been identified, including $\mathrm{Ca}^{2+}$, calcium-dependent protein kinases (CDPKs), 1,4,5-inositol triphosphate $\left(1,4,5-\mathrm{IP}_{3}\right)$, cyclic AMP (cAMP) and mitogen-activated protein kinases (MPK) [3, 4]. Moreover, gene expression changes are critical for plant adaptation to high-temperature stress. Recent research reports that transcription factors are important for plant responses to high temperature at the transcription level [5]. The latest report showed that high temperature-induced stomatal pores opening to facilitate leaf cooling requires blue light,

(c) The Author(s). 2020 Open Access This article is licensed under a Creative Commons Attribution 4.0 International License, which permits use, sharing, adaptation, distribution and reproduction in any medium or format, as long as you give appropriate credit to the original author(s) and the source, provide a link to the Creative Commons licence, and indicate if changes were made. The images or other third party material in this article are included in the article's Creative Commons licence, unless indicated otherwise in a credit line to the material. If material is not included in the article's Creative Commons licence and your intended use is not permitted by statutory regulation or exceeds the permitted use, you will need to obtain permission directly from the copyright holder. To view a copy of this licence, visit http://creativecommons.org/licenses/by/4.0/ The Creative Commons Public Domain Dedication waiver (http://creativecommons.org/publicdomain/zero/1.0/) applies to the data made available in this article, unless otherwise stated in a credit line to the data. 
suggesting the existence of an additional intracellular high-temperature response pathway in plants [6].

Phosphoinositide-specific phospholipase C (PLC) is a key enzyme in the phosphatidylinositol signalling system. Many species have multiple versions of PLC, and Arabidopsis thaliana has nine isoforms [7]. PLCs are involved in plant development and stresses responding. AtPLC2 in Arabidopsis thaliana regulates auxin levels in the vegetative and floral organs to influence the development of male and female gametophytes [8]. AtPLC2 also enhances tolerance to endoplasmic reticulum and drought stresses by regulating the biosynthesis of phosphatidic acid, salicylic acid and jasmonic acid $[9,10]$. The expression of TaPLC1 and TaPLC2 in wheat is induced by salt or drought [11]. Arabidopsis PLC3 participates in ABA responses in seed germination and stomatal closure. Seeds of the atplc3 mutant germinate slowly and are less sensitive to $\mathrm{ABA}$, preventing germination [12]. In addition, prior research in our lab has shown that overexpression of AtPLC9 and AtPLC3 in Arabidopsis substantially enhances heat tolerance [13, 14]. The rice genome includes four PLC genes, OsPLC1, OsPLC2, OsPLC3 and OsPLC4, on chromosomes 3, 5, 7 and 12, respectively. However, Arabidopsis and rice PLC family genes do not show a high degree of similarity, indicating that the genes have diverged during evolution [15]. OsPLC1 improves salt tolerance in rice [16]. In response to salt stress, OsPLC1 moves from the cytoplasm to the plasma membrane, where it increases cytosolic $\mathrm{Ca}^{2+}$, which controls sodium accumulation in the leaves, leading to improved salt tolerance [16]. OsPLC4 also enhances osmotic stress responses [17].

Rice (Oryza sativa) is an important staple food crop that are highly sensitive to high temperature stress at every growth stage such as seed germination, growth, and reproduction, particularly during the reproductive and grain-filling stages [18-21]. High temperatures have been shown to negatively affect rice yield and quality. Extremely high temperatures significantly decrease the grain yield by more than $50 \%$, even causing complete loss of harvest in rice plants [22]. High temperature affects the growth of rice at all stages. During flowering stage, heat stress impairs the pollen germination, anther dehiscence and pollen tube elongation, resulting in pollination failure [23]. Moreover, high temperature affects protein synthesis, transport, folding and assembly processes in rice grains and suppresses of genes expression related to starch biosynthesis [24]. It is necessary to improve the adaptability of rice to cope with the high temperature stress. However, the genetic basis for heat tolerance or adaptability to heat stress in crop plants, including rice, is poorly understood.

Heterologous transformation of genes identified in Arabidopsis into monocot crop species such as rice can help elucidate gene function and identify genes capable of conferring adaptive traits, such as heat tolerance, in crops. In this study, we aimed to determine similarities and differences in the transduction of heat-shock signals through PLC in rice and Arabidopsis and to determine if AtPLC9 can be used to enhance the heat tolerance of cultivated rice. We cloned AtPLC9 and ectopically expressed it in the Asian rice cultivar 'Changyou No. 1'. Using high-throughput RNA sequencing (RNA-seq), we analysed transcriptome-level changes induced by expression of AtPLC9 in rice under normal and heat stress conditions. We observed that rice heterologously expressing AtPLC9 exhibited much higher expression of heat shock factors (OsHSFAs) and calcium ion and calmodulin related genes than wild type plants when exposed to HS. Our results suggest that AtPLC9 may enhance heat tolerance in rice and Arabidopsis through similar mechanisms. AtPLC9 conferred heat resistance both in monocots and dicots and these results implied that important elements of the HS response are conserved between monocots and dicots. Our results enhance understanding of the molecular mechanisms of heat-shock response in plants and demonstrate that AtPLC9 is an important and valuable resource to improve heat resistance in molecular breeding programs. More broadly, this study highlights the potential for heterologous transformation to assist in molecular breeding.

\section{Results}

\section{Heterologous expression of AtPLC9 improves heat tolerance in rice}

To assess the heat-tolerance potential of AtPLC9 in rice, we heterologously expressed AtPLC9 coding region driven by $35 \mathrm{~S}$ promoter, as described in our previous study [14] in 'Changyou No. 1' to obtain three independent T4 homozygous transgenic rice lines: OE-7, OE-9 and OE-10. All three lines exhibited higher AtPLC9 expression levels than the wild type (WT), indicating that they were expressing AtPLC9 (Supplementary Fig. 1). We then subjected the three lines to HS treatment to examine their phenotypes. Seven-day-old seedlings grown at $28^{\circ} \mathrm{C}$ were treated for $15 \mathrm{~min}$ at $45^{\circ} \mathrm{C}$ or were shifted to $37^{\circ} \mathrm{C}$ for $30 \mathrm{~min}$ and then returned to normal conditions $\left(28^{\circ} \mathrm{C}\right)$ for $2 \mathrm{~h}$ before again subjecting to $45^{\circ} \mathrm{C}$ for $45 \mathrm{~min}$ to determine basal thermotolerance and acquired thermotolerance, respectively. Seedlings were then grown for another 7 days at $28^{\circ} \mathrm{C}$ before we analysed their phenotype, took pictures and calculated their survival rates, electrolyte leakage and chlorophyll content. Significantly more seedlings expressing AtPLC9 survived compared to the WT in both basal and acquired thermotolerance tests (Fig. 1a, b and Supplementary Fig. 2A, B). WT seedlings exhibited severe wilting and chlorosis and few seedlings survived. In comparison, 


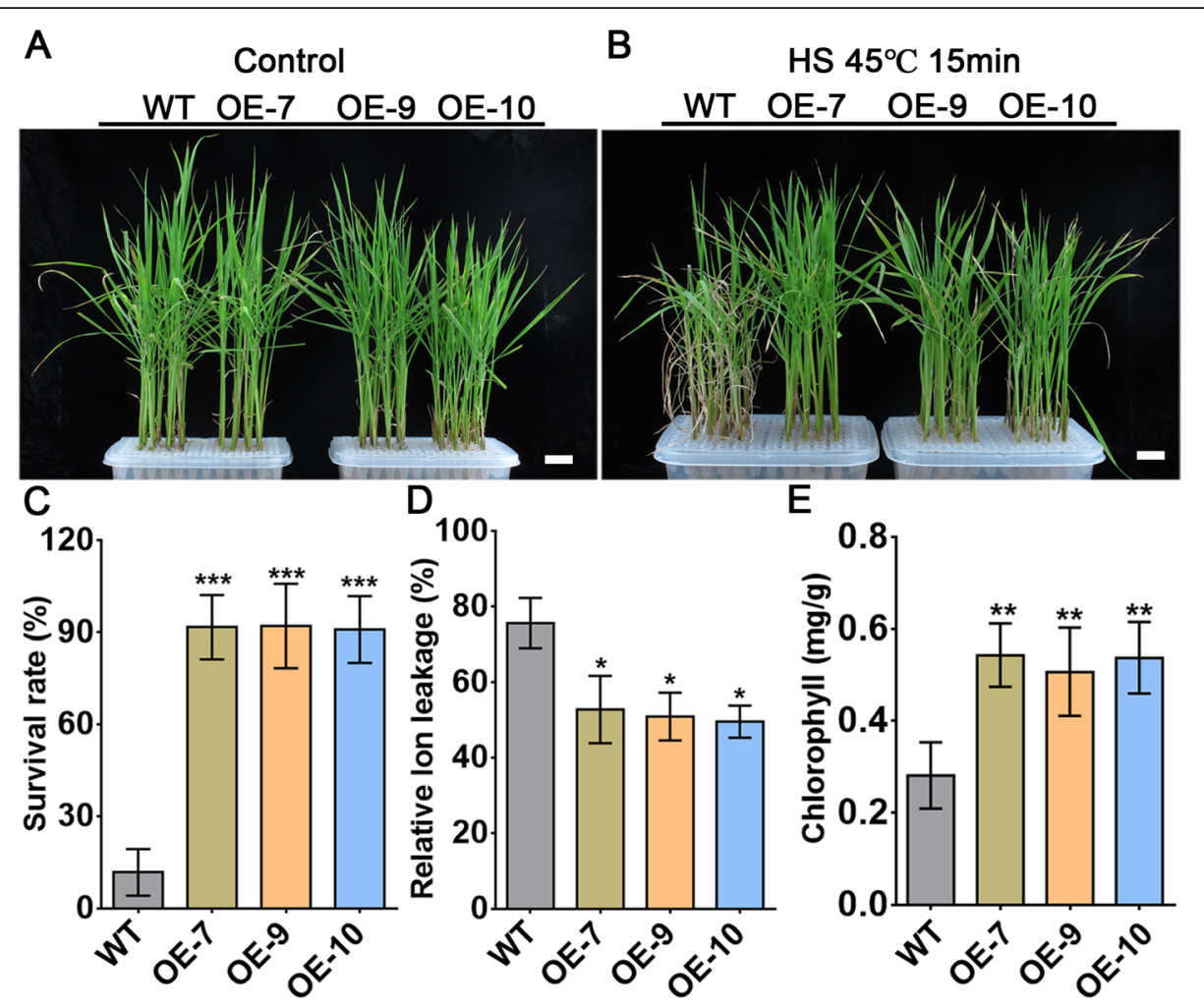

Fig. 1 Expression of AtPLC9 improves the basic tolerance of rice. a. Fourteen-day-old rice (WT and transgenic lines OE-7, OE-9 and OE-10) grown in Hoagland's solution under normal conditions $\left(28^{\circ} \mathrm{C}\right)$ were used as controls. b. Seven-day-old rice (WT, OE-7, OE-9 and OE-10) grown in Hoagland's solution under normal conditions $\left(28^{\circ} \mathrm{C}\right)$ were shifted to $45^{\circ} \mathrm{C}$ for $15 \mathrm{~min} \mathrm{HS}$ treatment and then returned to normal conditions $\left(28^{\circ} \mathrm{C}\right)$ for another 7 days. c. Survival rate of WT, OE-7, OE-9 and OE-10 after HS. Each value is the mean $\pm \mathrm{SE}, n=3$. $\mathbf{d}$. Relative ion leakage of plants after HS treatment. Each value is the mean $\pm \mathrm{SE}, n=3$. e. Chlorophyll content of seedlings after $\mathrm{HS} ; 0.1 \mathrm{~g}$ of fresh tissue was used in each experiment. Each value is the mean $\pm \mathrm{SE}, n=3$. Student's $t$-test was used to calculate $P$ values. ${ }^{* * *} p<0.001,{ }^{* *} p<0.01,{ }^{*} p<0.05$, statistics was analysed between OE-7/- 9/- 10 vs WT. Bars (a and $\mathbf{b})=1 \mathrm{~cm}$

the three heterologous expression lines had survival rates significantly exceeding that of the WT (Fig. 1c and Supplementary Fig. 2C). Correspondingly, HS-induced electrolyte leakage, an indicator of plasma membrane damage, was dramatically lower in the AtPLC9-expressing transgenic plants compared with that in WT plants under HS conditions (Fig. 1d and Supplementary Fig. 2D). The chlorophyll content in three heterologous expression lines was significantly higher than that in WT plants after HS treatment (Fig. 1e and Supplementary Fig. 2E). These results suggest that the heterologous expression of AtPLC9 greatly improves the heat tolerance of rice.

To examine whether expression of AtPLC9 confers tolerance to other abiotic stresses, we performed salt, drought and cold stress tests on the transgenic lines. After a 7-day treatment with $200 \mathrm{mM} \mathrm{NaCl}$, the WT and the three transgenic lines all exhibited withered leaves (Supplementary Fig. 3A). All plants died following salt treatment for 14 days, and we observed no difference between WT and the three heterologous expression lines in their response to salt stress (Supplementary Fig. 3B).
These results suggest that heterologous expression of AtPLC9 does not confer enhanced tolerance to salt stress.

We simulated drought stress by treating plants with different concentrations of mannitol. After 2 days growing on $350 \mathrm{mM}$ mannitol, both the WT and the three transgenic lines exhibited withered leaves and were smaller than control plants exposed to $0 \mathrm{mM}$ mannitol (Supplementary Fig. 4A). After 10 days of treatment with $350 \mathrm{mM}$ mannitol, the WT and heterologous expression lines all exhibited dry and withered leaves (Supplementary Fig. 4B). There were no statistically significant differences in survival rate between the WT and the three transgenic lines, suggesting that heterologous expression of AtPLC9 in rice does not affect drought tolerance.

Previous research has shown that OsPLDa1 improves the response of rice to cold stress by increasing the cytoplasmic concentration of $\mathrm{Ca}^{2+}$, thus increasing the transcriptional activity of COLD-RESPONSIVE C REPEAT/DEHYDRATION-RESPONSIVE ELEMENT BINDING 1 (OsDREB1) [25]. We therefore wondered whether AtPLC9 could also 
improve the cold tolerance of rice. To address this question, we subjected the WT and the three heterologous expression lines to cold stress. Seedlings grown for 7 days at $28^{\circ} \mathrm{C}$ were transferred to $4{ }^{\circ} \mathrm{C}$ for 7 days of cold treatment. The WT and heterologous expression lines exhibited similar restrictions in plant height under $4{ }^{\circ} \mathrm{C}$ (Supplementary Fig. 5A and $5 \mathrm{~B}$ ), which suggested that AtPLC9 does not affect cold tolerance in rice. In conclusion, the heterologous expression of AtPLC9 specifically improves the tolerance of rice to heat stress, but not other abiotic stresses.

\section{Heat stress triggers transcriptome reprogramming in AtPLC9 heterologous expression lines}

To determine how AtPLC9 coordinates the response to HS in rice, we assessed the transcriptome of heterologous expression lines following HS treatment. We first assessed the expression level of heat shock factors (HSFs; Os03g0745000, Os04g0568700, Os08g0546800), Multiprotein-bridging factor 1c (MBF1 Os06g0592500) and heat shock proteins (HSPs OS01g0136000 and Os03g0266900). Similar AtPLC9 expression levels were detected in the three transgenic rice lines (OE-7, OE-9, and OE-10), and they also displayed same phenotype; therefore, only OE-7 was used in subsequent experiments. Before and after HS, none of these genes showed significant differences in their expression levels between OE7 and WT (Supplementary Fig. 6). This result indicated that AtPLC9 may regulate the HS response through other genes, so we conducted Illumina high-throughput sequencing to search for other regulatory pathways.

We compared the expression profiles of four samples, designated WT-CK (wild type without HS), WT-HS (wild type after a 15-min HS), OE-7-CK (AtPCL9-expressing line 7 without HS) and OE-7-HS (AtPCL9-expressing line 7 after a 15-min HS). Each sample contained three biological replicates, from which approximately 879.8 million 150-bp paired-end reads were generated (Supplementary Table 1). After discarding the low-quality reads, 63.6 to 84.2 million reads were kept for subsequent analysis. More than $88 \%$ of reads containing no more than three mismatches were mapped to the reference genome, and over $73 \%$ of reads showed unique alignments (Supplementary Table 1). Both principal component analysis (PCA) (Fig. 2a) and hierarchical clustering demonstrated high reproducibility among biological replicates (Fig. 2b). Furthermore, PCA revealed a clear divergence in expression profiles between the normal and HS conditions (Fig. 2a). Heat stress amplified the difference in expression profiles between OE-7 and WT (Fig. 2a), which was consistent with our phenotypic observations.

We then performed differential expression analysis between normal and HS conditions for the two genotypes, designated WT:HS/CK and OE-7:HS/CK. Using fold change $\geq 2$ and False Discovery Rate (FDR) $<0.05$ as a cutoff, we identified 1578 differentially expressed genes
(DEGs) in the WT:HS/CK comparison, of which 932 and 648 genes were upregulated and downregulated by HS, respectively (Fig. 3a; Supplementary Dataset 1). The number of DEGs (2435), upregulated genes (1181) and downregulated genes (1254) in OE-7:HS/CK were all higher than in WT:HS/CK (Fig. 3a; Supplementary Dataset 2), suggesting that more extensive and dynamic transcriptome reprofiling occurred in OE-7 following HS. Venn diagram results revealed that 1464 DEGs (60.12\%), 756 upregulated genes (64.01\%) and 756 downregulated genes $(60.29 \%)$ specifically existed in the OE-7: HS/CK comparison (Fig. 3b), which were designated as OE-7-specific genes. We next performed gene ontology (GO) term enrichment analysis of genes showing altered expression levels in WT and OE-7 after HS. Known HS GO terms were significantly enriched among the genes upregulated by heat in both WT and OE-7, including 'response to heat' (GO:0009408), 'protein folding' (GO: 0006457) and 'response to osmotic stress' (GO:0006970) (Supplementary Fig. 7). GO terms enriched among genes downregulated in both WT and OE-7 exposed to HS included 'transport' (GO:0006810), 'brassinosteroid homeostasis' (GO:0010268) and 'phosphorylation' (GO: 0016310) (Supplementary Fig. 7). Some GO terms were significantly enriched only in the OE-7-specific HSupregulated genes, such as 'regulation of response to stress' (GO:0080134), 'regulation of metabolic process' (GO:0019222) and 'regulation of gene expression' (GO: 0010468) (Supplementary Fig. 7). To further evaluate the overall trend of expression change, we calculated the Pearson correlation coefficient between the fold change of gene expression levels in WT and OE-7 after HS and the fold change of gene expression levels under unstressed conditions. The ratios of expression change for genes related to 'response to heat' (GO:0009408) in OE7 correlated strongly with the corresponding ratios in WT ( $\mathrm{R}=0.97$; Supplementary Fig. 8). However, the expression changes of the remaining genes were only moderately positively correlated $(\mathrm{R}=0.58)$ (Supplementary Fig. 8). These results suggest that the transcriptome reprogramming induced by $\mathrm{HS}$ in $\mathrm{OE}-7$ is similar to, but distinguishable from, the corresponding transcriptome changes in the WT. Collectively, the above results suggest that a more extensive and dynamic transcriptome reprofiling occurs in transgenic plants after heat stress, and that this widespread transcriptome change may account for the heat tolerance conferred by heterologous expression of AtPLC9.

Rice heterologously expressing AtPLC9 under HS exhibits significantly higher expression of transcription factors and $\mathrm{Ca}^{2+} /$ calmodulin (CaM) signal transduction pathway genes We also compared the transcriptomes between OE-7 and WT under normal conditions (CK:OE-7/WT) and 


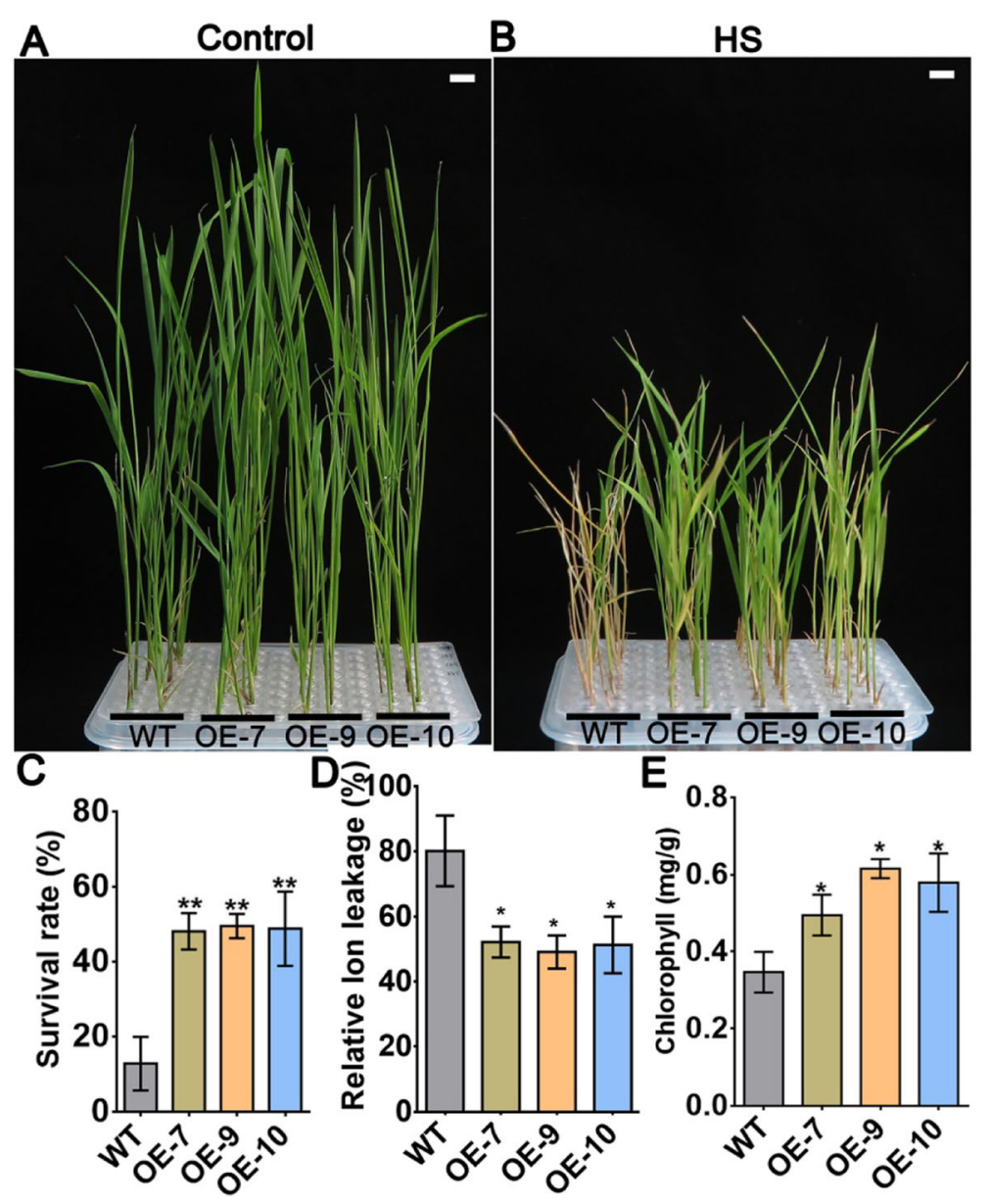

Fig. 2 Expression of AtPLC9 in rice improves acquired thermotolerance. a. 14-d-old rice (WT and transgenic lines OE-7, OE-9 and OE-10) grown under normal conditions $\left(28^{\circ} \mathrm{C}\right)$ were used as controls. White bar $=1 \mathrm{~cm}$. b. 7-d-old rice (WT, OE-7, OE-9 and OE-10) grown under normal conditions $\left(28^{\circ} \mathrm{C}\right.$ ) were shifted to $37^{\circ} \mathrm{C}$ for $30 \mathrm{~min}$ and then returned to normal conditions $\left(28^{\circ} \mathrm{C}\right)$ for $2 \mathrm{~h}$ before $45^{\circ} \mathrm{C} \mathrm{HS}$ treatment for 45 min. After the treatment, the seedlings were returned to $28^{\circ} \mathrm{C}$ for another $7 \mathrm{~d}$. White bar $=1 \mathrm{~cm}$. c. The survival rate of WT, OE-7, OE-9 and OE-10 after $H S$. Each value is the mean $\pm S E, n=3$. d. Relative ion leakage quantification of plants after $H S$ treatment. Each value is the mean $\pm S E, n=3$. e. Chlorophyll content of the seedlings after HS; $0.1 \mathrm{~g}$ of fresh tissue was used in each experiment. Each value is the mean \pm SE, $n=3$. Student's $t-$ test was used to calculate the $P$ value. ${ }^{* *}$ indicates $P$ value $<0.01,{ }^{*}$ indicates $P$ value $<0.05$, statistics was analysed between OE-7/- 9/- 10 vs WT

under HS (HS:OE-7/WT). We detected 384 and 782 DEGs under normal and HS conditions, respectively (Fig. 3a; Supplementary Datasets 3 and 4). Consistent with the PCA (Fig. 2), the number of DEGs under HS was double that under unstressed conditions. Intriguingly, 532 genes showed upregulated expression levels in OE-7 under HS, which was substantially higher than the number of corresponding genes (143) under unstressed conditions (Fig. 3a). However, the number of downregulated genes were similar between the HS (250) and CK (241) comparisons (Fig. 3a). Compared with the CK:OE/WT comparison, we identified 629 DEGs, 460 upregulated genes and 220 downregulated genes specifically existing in the HS:OE/WT comparison (Fig. 3b), representing $80.43,86.47$ and $88.00 \%$. These genes were designated as HS-specific genes. GO term enrichment analysis revealed that those genes upregulated in the HS:
OE-7/WT comparison were primarily enriched in the terms 'regulation of response to stress' (GO:0080134), 'regulation of metabolic process' (GO:0019222) and 'regulation of gene expression' (GO:0010468), which were specifically enriched in the OE-7 HS-induced gene set as well (Supplementary Fig. 7).

We detected 272 DEGs, 250 upregulated genes and 99 downregulated genes overlapping between OE-7- and HS-specific genes (Fig. 3b), which we identified as the potential targets regulated by AtPLC9 during HS response in rice. We conducted GO term enrichment analysis on these genes to examine the function of targets potentially regulated by AtPLC9 during HS. No specific GO terms were enriched in downregulated target genes (Fig. 4). Upregulated target genes and DEGs were enriched in the same GO terms (Fig. 4). Since AtPLC9 is a positive regulator of the HS response [14], the 250 


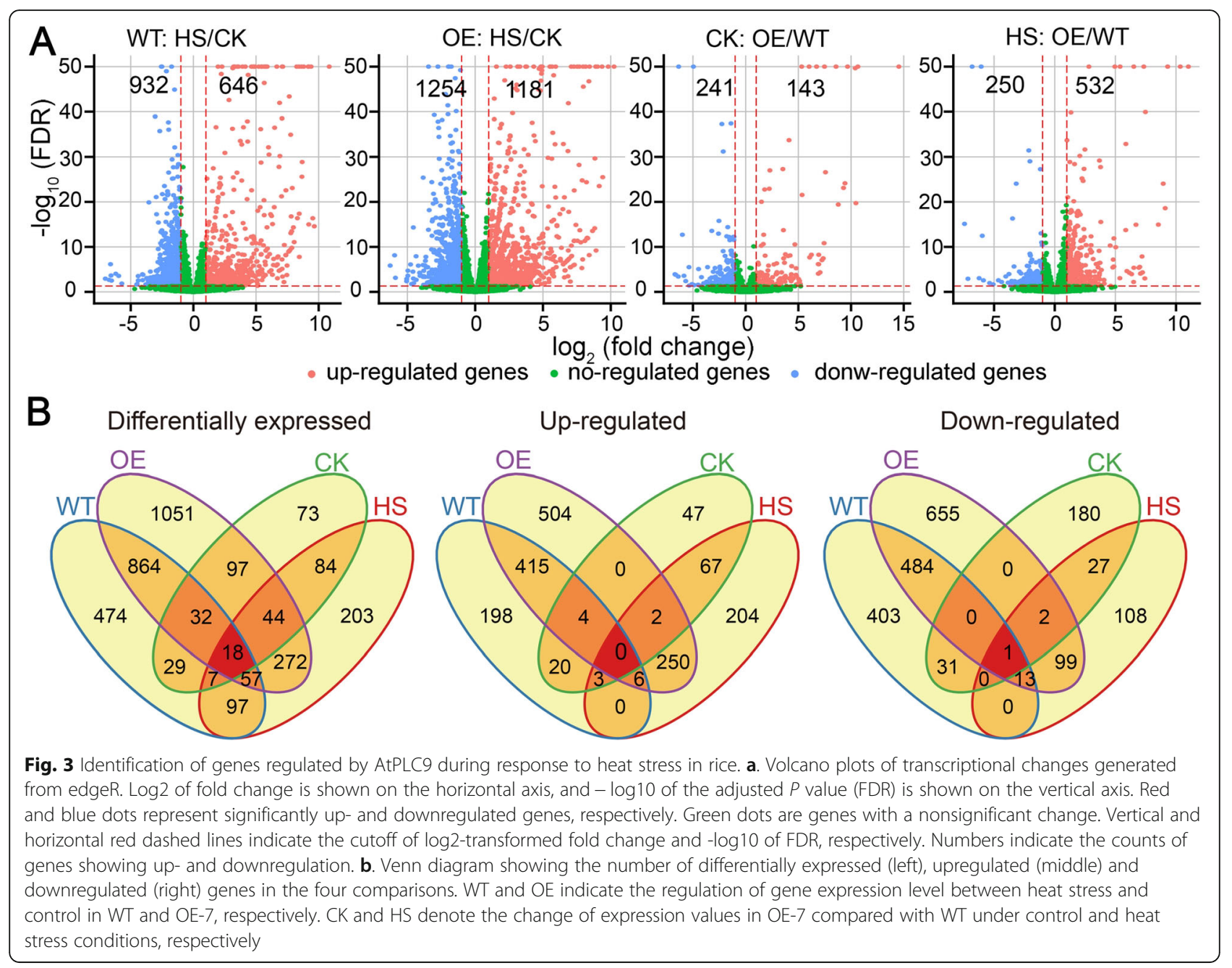

upregulated target genes were subjected to more detailed analysis (Supplementary Dataset 5). These upregulated target genes were enriched in the GO terms 'regulation of transcription' (GO:0045449) and 'regulation of gene expression' (GO:0010468) (Fig. 4), which suggested that some of these target genes may be transcription factors (TFs) or transcription regulators (TRs). Using the iTAK pipeline [26], we identified and classified 52 putative TF (51) and TR (1) members from 13 families among these target genes, including WRKY (11; Os03g0335200), AP2/ ERF (9; Os02g0781300), Tify (7; Os03g0180900), MYB (6; Os09g0401000) and HSF (1; Os02g0527300). The $\mathrm{Ca}^{2+} /$ calmodulin $(\mathrm{CaM})$ signal transduction pathway has a significant role in the heat stress response [14]. From these 250 upregulated target genes, we identified 17 genes annotated as functioning in this pathway, such as calcium-dependent protein kinases (CDPKs). To test the accuracy of our transcriptome-level analysis, we used quantitative RT-PCR to validate the expression levels of six randomly selected upregulated target genes, including five TFs and one CDPK (Os08g0540400). Consistent with the RNA-seq data, RT-qPCR revealed that the expression levels of these six genes were substantially elevated in OE-7, OE-9 and OE-10 compared with those in the WT after HS (Fig. 5 and Supplementary Fig. 9). Together, these finding suggest that the expression of particular TFs and $\mathrm{Ca}^{2+} / \mathrm{CaM}$ pathway-related genes was specifically upregulated by $\mathrm{HS}$ in the AtPLC9-expressing lines, which may contribute to the improved heat stress tolerance observed in these lines.

\section{Discussion}

In this study, we transformed the Arabidopsis gene AtPLC9 into the rice cultivar 'Changyou No. 1', identified its heat-tolerant phenotype, and analysed the transcriptome-level changes induced by AtPLC 9 under normal and HS conditions. This study contributes to our understanding of the biological function, molecular mechanism and heat-tolerance mechanism of AtPLC9 in monocotyledons as well as dicotyledons. We conclude that AtPLC9 is a valuable gene resource conferring heat 


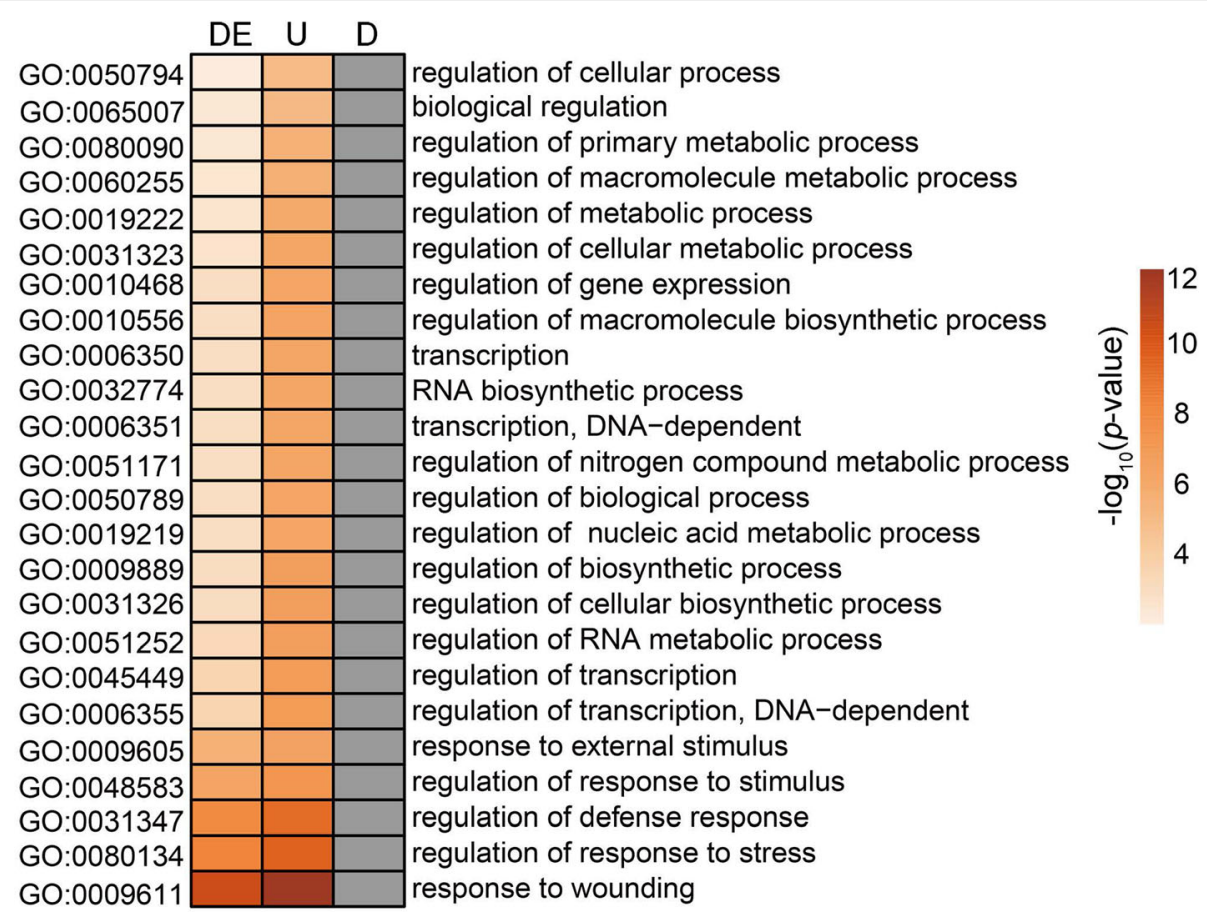

Fig. 4 Enriched GO terms among candidate genes regulated by AtPLC9 during response to heat. The colour in each cell indicates -log10transformed $P$ value of the $\mathrm{GO}$ enrichment tested by hypergeometric test with Bonferroni correction; blank cells are not significant. DE, $U$ and $D$ indicate differentially expressed, upregulated and downregulated genes, respectively

resistance both in monocots and dicots, setting the stage for its use to improve crop heat tolerance via molecular breeding programs.

\section{AtPLC9 confers heat tolerance to monocotyledons as well as dicotyledons}

Our previous studies have shown that AtPLC9 and AtPLC3 both enhance heat tolerance in Arabidopsis thaliana $[13,14,27]$. In this study, we observed that rice heterologously expressing AtPLC9 also exhibits improved heat tolerance. The survival rate of AtPLC9-expressing lines exposed to $45^{\circ} \mathrm{C}$ was eight times more than that of the WT. These results suggest that components of the HS response involving AtPLC9 are conserved between monocotyledons and dicotyledons.

When rice is exposed to salt stress, OsPLC1 moves from the cytoplasm to the cell membrane where it regulates $\mathrm{Ca}^{2+}$ responses [16]. In this study, expression of OsPLC1 and OsPLC2 was increased after HS treatment. However, OsPLC3 and OsPLC4 were not significantly upregulated by heat stress (Supplementary Fig. 10). More research is necessary to identify the molecular mechanisms underlying how OsPLCs respond to HS. Assessing how endogenous OsPLCs function after HS will help us to more fully understand PLC regulation of thermotolerance in rice.
Based on our previous research, we proposed that plants respond to $\mathrm{HS}$ through the calcium-calmodulin heat-shock pathway. The calcium-calmodulin pathway is now recognized as a major channel for heat-shock response $[2,28,29]$. Following heat shock, a signal is conveyed from the cell membrane to intracellular targets. Heat shock increases the concentration of cytoplasmic calcium, which in turn increases the expression of CALMODULIN 3 (CaM3). CAM-BINDING PROTEIN KINASE 3 (CBK3) regulates the phosphorylation status and binding activity of heat shock factors (HSFs) to heat shock elements (HSEs), which ultimately regulates thermotolerance by promoting or inhibiting the expression of HSPs $[3,4,30-32]$. In animal cells, $\mathrm{IP}_{3}$ binds to the $\mathrm{IP}_{3}$ receptor on the endomembrane system, which releases $\mathrm{Ca}^{2+}$ from intracellular stores [33, 34]. However, it is not clear whether PLC in plants functions as it does in animals. Preliminary studies have shown that within $1 \mathrm{~min}$ following heat shock, the level of $\mathrm{IP}_{3}$ rapidly increases. After $3 \mathrm{~min}, \mathrm{IP}_{3}$ levels reach their maximum of 2.5 times the level prior to heat shock [22]. In addition, $\mathrm{IP}_{3}$ can induce the expression of HSP18.2 promoter: GUS in the absence of heat shock [3]. The phospholipase C-specific inhibitor U73122 limits the heat-shock-induced increase in $\mathrm{IP}_{3}$ and $\mathrm{Ca}^{2+}$ in Arabidopsis thaliana suspension cells [3]. AtPLC9 generates $\mathrm{IP}_{3}$ in the cell following $\mathrm{HS}$, stimulating the release of 


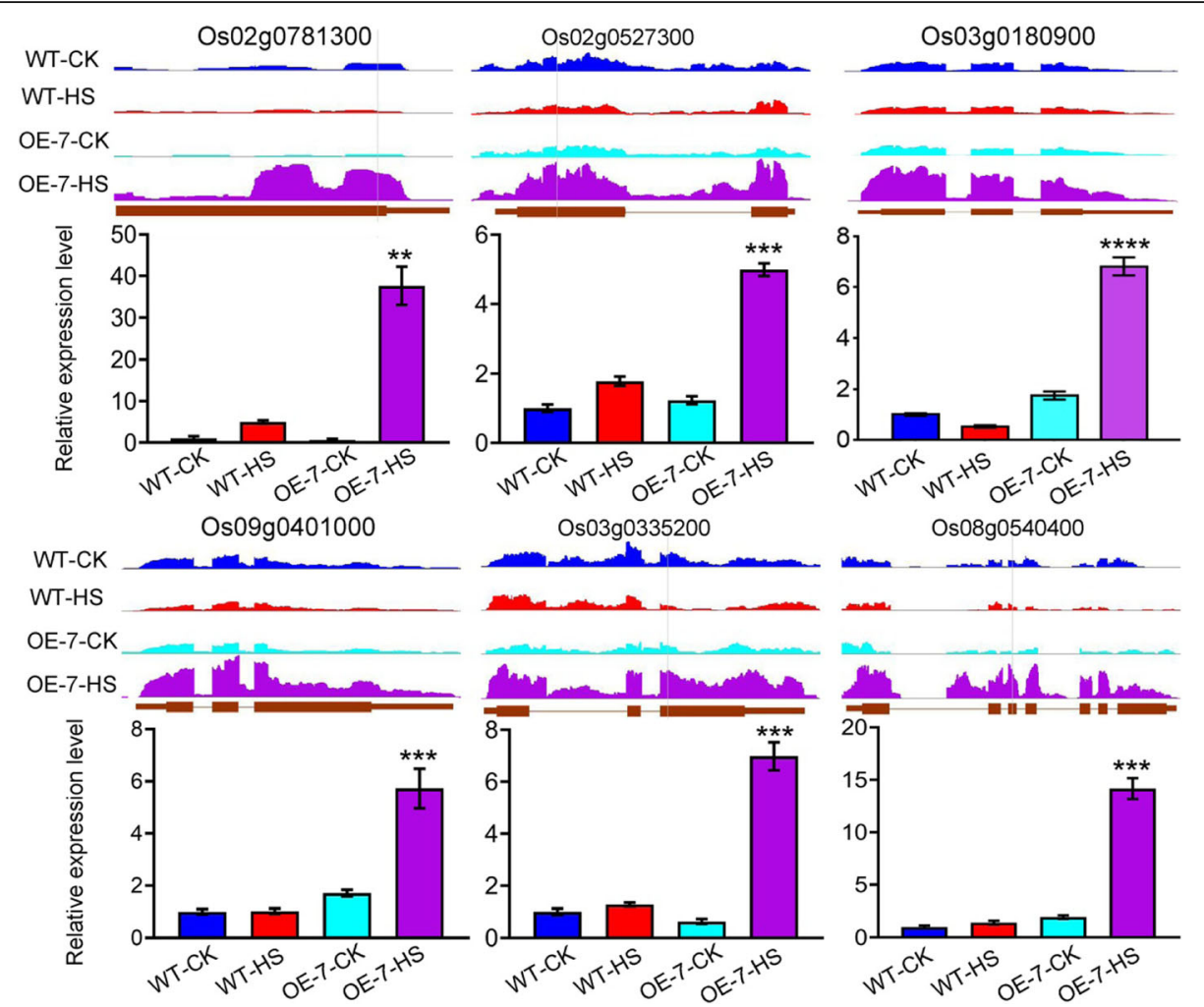

Fig. 5 Validation of RNA-seq data using real-time quantitative PCR for six potential AtPLC9 target genes. RNA-seq signals visualized using Integrated Genome Browser (IGB; http://www.bioviz.org) and real-time quantitative PCR quantification of the RNA levels for six candidate AtPLC9regulated genes in wild-type (WT) and OE-7 plants before (CK) and after HS treatment. Values for RT-qPCR results are means \pm SE $(n=3)$. Student's $t$-test was used to calculate $P$ values. ${ }^{* * *} P<0.0001$, ${ }^{* * *} P<0.001$, ${ }^{* *} P$ value $<0.01$, statistics was analysed between OE-7-HS vs WT-HS

$\mathrm{Ca}^{2+}$ from intracellular stores and promoting the transmission of heat-shock signals [14].

In our current study, we observed that rice heterologously expressing AtPLC9 exhibited much higher expression of OsHSFAs and calcium ion and calmodulin related genes than the WT when exposed to HS. These results suggest that AtPLC9 may enhance heat tolerance in rice and Arabidopsis through similar mechanisms and imply that important elements of the HS response are conserved between monocots and dicots.

\section{AtPLC9 specifically confers tolerance to heat shock, but not to other abiotic stresses}

AtPLC4, 5, and 7 in Arabidopsis are upregulated under salt or drought stress [35]. Recent research has shown that OsPLC1 regulates salt tolerance in rice by increasing the cytoplasmic concentration of $\mathrm{Ca}^{2+}$. Following salt stress, OsPLC1 moves from the cytoplasm to the plasma membrane, where it increases cytoplasmic $\mathrm{Ca}^{2+}$, which regulates $\mathrm{Na}^{+}$accumulation in leaves and improves salt tolerance [16]. Prior research in our laboratory indicates that AtPLC9 regulates the concentration of cytoplasmic $\mathrm{Ca}^{2+}$ through the second messenger $\mathrm{IP}_{3}$ to influence the expression of HSF and HSP and enhance the heat tolerance of Arabidopsis [13, 14]. AtPLC4 responds to salt stress, and its mutant also displays a hyposensitivity phenotype [36].

In our current study, we exposed rice heterologously expressing AtPLC9 to salt and drought stress and observed no significant difference in growth, phenotype or survival rate between transgenic lines and the WT. These results suggest that AtPLC9 functions differently from OsPLCs in rice, and that the former does not affect the sensitivity of rice to salt or drought. Prior research has shown that OsPLD $\alpha$ l responds to cold stress [25]. Yet, after cold stress treatment, we observed no difference in growth between the WT and rice heterologously expressing AtPLC9, suggesting that AtPLC9 cannot improve cold tolerance in rice. We conclude that heterologously expressing AtPLC9 only confers greater tolerance to heat, but not to other abiotic stresses. Cross tolerance is a critical strategy for plant to adapt environmental stress. For example, heat stress tolerance also improves other stress in Arabidopsis [37]. However, in this study, we found that AtPLC9 transgenic rice specifically response to heat stress (Fig. 1 and Supplemental Fig. 2). We speculate that there may be two reasons for this. 
First, the generation of cross adaption was sequentially regulated. Second, heat stress signal transduction may be partially independent to other stress. This result may also provide an experimental clue for study the mechanism of cross adaption in the future.

\section{Dynamic changes in transcriptional levels may be an important strategy for monocots and dicots to respond to short-term high-temperature stress}

Compared to the WT, rice plants heterologously expressing AtPLC9 exhibited a greater number of transcriptional changes, including changes in expression levels of TFs, when exposed to HS, which suggests that the regulation of gene transcription is an important strategy for plants to respond to short-term high-temperature stress.

We also observed abnormal expression of $\mathrm{Ca}^{2+}$-related signalling pathway genes in AtPLC9-expressing transgenic rice, suggesting that $\mathrm{Ca}^{2+}$ signalling may be an important pathway for plants to respond relatively quickly to shortterm stress. Six marker genes were chose and the expressions were analyzed. These six genes response to environmental factors as reported in the plant Regulomics database (http://bioinfo.sibs.ac.cn/plant-regulomics/index. php) [38], an intergraded on-line database. The transcriptomic comparisons result indicated that the expressed levels of all these six genes can be regulated by biotic or abiotic stresses in previous studies. The result of study (GSE33204) indicated that OsO2g0527300 (OsHsfA3) and Os03g0335200 (OsWRKY44) showed upregulation, and showed downregulation in 30-day-old leaves of rice after $6 \mathrm{H}$ high temperature treatment [39]. However, these three genes did not show alternated expression level in WT induced by heat stress in our study, which might be attributed to the different genotype, development stage, tissue or treatment condition. The expression of the remained genes can be induced or repressed by other biotic or abiotic stresses significantly. For example, significantly induced by salt treatment and highly expressed in spikelet and developing seeds, Os08g0540400 (OsCPK21) can improve the salt tolerance of rice [40]. Many factors affect the hightemperature stress response, beyond the modulation of gene expression. Therefore, it also may be interesting to look at the heat-tolerant phenotype induced by AtPLC9 expression histologically.

\section{Feasibility of heterologous transformation for assessing gene function and conferring adaptive traits}

Heterologous transformation helps the study of gene function and can be used to improve crop yield. This study demonstrated that AtPLC9 can also function in the monocotyledon rice. Heterologous transformation has broad applications in agricultural production, including the transfer of genes between wild and cultivated species, and between different families. However, researchers turning to heterologous transformation must consider the genetic diversity and evolutionary relationships between species. Heterologous genes may not retain their functions if transferred between species that have diverged too far.

We show that the heat-related gene AtPLC9 from Arabidopsis functions well in rice in defending against heat shock. This contributes to our understanding of the molecular mechanisms of the heat-shock response in plants. Further exploration of AtPLC9 in other plant species could provide us more information regarding the functional conservation of elements in $\mathrm{Ca}^{2+} / \mathrm{CaM}$ HS signal transduction pathway. In addition, our results highlight that heterologous transformation has the potential to assist with molecular breeding both in monocots and dicots.

\section{Methods}

Plant materials and growth conditions

Rice seedlings were grown hydroponically in an illuminated incubator with $16 \mathrm{~h}$ light $\left(28^{\circ} \mathrm{C}\right) / 8 \mathrm{~h}$ dark $\left(28^{\circ} \mathrm{C}\right)$ photoperiod. Heat treatment was performed at $45^{\circ} \mathrm{C}$ in a water bath. The seedlings grown in liquid medium for 7 days were moved in water bath for $45^{\circ} \mathrm{C}$ HS treatment. Meanwhile, the control plants was put into water at $28^{\circ} \mathrm{C}$ for same time. Cold treatment was performed at $4{ }^{\circ} \mathrm{C}$ in a low temperature incubator. For salt treatment, plants were exposed to $0 \mathrm{mM}, 50 \mathrm{mM}, 100 \mathrm{mM}, 150 \mathrm{mM}$ or $200 \mathrm{mM} \mathrm{NaCl}$. To simulate drought, plants were exposed to $0 \mathrm{mM}, 150 \mathrm{mM}, 250 \mathrm{mM}$ or $350 \mathrm{mM}$ mannitol. Untreated seedlings were used as controls.

\section{Measurement of chlorophyll content and ion leakage}

Chlorophyll content was determined as described by [41].

After HS treatment, $0.1 \mathrm{~g}$ seedlings was harvested in 4 $\mathrm{ml}$ deionized water. Conductivity of the solution was determined using a conductivity meter (METTLER TOLEDO FE38). Relative ion leakage was calculated according to the method of [25].

\section{Gene expression analysis}

Total RNA was isolated from 14-day-old seedlings then translated into cDNA and used as template for quantitative PCR. AtPLC9 transcript abundance in WT and transgenic plants was determined by RT-PCR using primers amplifying the AtPLC9 coding region (Supplementary Table 2). OsTUBULIN was used as a control. Quantification of the expression level for AtPLC9-regulated genes in WT and OE-7 plants was performed by real-time quantitative PCR. Expression of Os02g0781300, Os02g0527300, Os01g0968800, OsO 9g0401000, Os03g0335200, Os08g0540400 and Os02g0781 300 was analysed using primers given in Supplementary Table 2. Quantification of expression levels of OSPLC genes was also performed by real-time quantitative PCR using primers described by [16]. 


\section{RNA sequencing analysis}

Total RNA was isolated from 14-day-old seedlings using a TRIzol kit (Invitrogen). Paired-end sequencing libraries with an average insert size of $200 \mathrm{bp}$ were prepared with a TruSeq RNA Sample Preparation Kit version2 (Illumina) and sequenced on a HiSeq Xten according to the manufacturer's standard protocols. FastQC (http://www.bioinf ormatics.babraham.ac.uk/projects/fastqc/) was initially run to assess the overall quality of all sequenced reads. Poor-quality reads were filtered out using Sickle with the parameters pe-mode; -t sanger-q $20-150$ (https://github. com/najoshi/sickle). The remaining high-quality reads were mapped to the Oryza sativa Japonica reference genome (http://plants.ensembl.org/Oryza_sativa/Info/Index) using TopHat v2.09 with the parameters "-N 3-read- editdist 3-segment-mismatches 1 -p 20 -r 0 -g 20 --microexon-search-b2-D 20-b2- R3-no-coverage-search", and only reads showing unique alignments were retained for the following analysis. The cuffquant and cuffnorm components of cufflinks (2.2.1) with default parameters were used for normalization and estimation of gene expression levels. Only genes with a mean expression level of 1 read per kilobase of transcript per million mapped reads (FPKM) in at least one sample were considered expressed genes and used to calculate the Pearson correlation coefficient and PCA for replicates of each sample. HTseq software (http://www-huber.embl.de/ users/anders/HTSeq/doc/overview.html) was used to count the read counts mapped to each of the genes. The Bioconductor package "edgeR" was used for differential expression analysis [42]. Only genes with FDR $<0.05$ and absolute value of $\log 2$ (fold change) $\geq 1.0$ were considered DEGs in subsequent analysis. GO term enrichment was analysed with Singular Enrichment Analysis provided by AgriGO [43] using a hypergeometric test, with FDR < 0.05 as cutoff. Rice GO terms downloaded from EnsemblPlants (http://plants.ensembl.org/index.html) were used as background references. The RNA-seq data were deposited in the NCBI SRA database (http://www.ncbi.nlm.nih.gov/ sra) with BioProject number PRJNA597792.

\section{Supplementary Information}

Supplementary information accompanies this paper at https://doi.org/10. 1186/s12870-020-02709-5.

Additional file 1: Supplemental Data Set 1: List of genes differentially expressed between HS treatment and control of WT.

Additional file 2: Supplemental Data Set 2: List of genes differentially expressed between HS treatment and control of OE7.

Additional file 3: Supplemental Data Set 3: List of genes differentially expressed between OE7 and WT without HS treatment.

Additional file 4: Supplemental Data Set 4: List of genes differentially expressed between OE7 and WT after HS treatment.

Additional file 5: Supplemental Data Set 5: List and function annotation of putative targeted genes.
Additional file 6: Supplemental Data Set 6: Transcriptomic

comparison results of six selected AtPLC9 targets generated from Plant Regulomics database.

Additional file 7.

\section{Abbreviations}

HS: Heat shock; CDPKs: Calcium-dependent protein kinases; 1,4,5-IP $:$ 1,4,5inositol triphosphate; PLC: Phosphoinositide-specific phospholipase C; CaM: Calmodulin

\section{Acknowledgements}

Thanks to Yixin Li for planting rice seedlings in the field. Thanks to Hebei YINCAIJIHUA program organized by Hebei Association for Science and Technology.

\section{Authors' contributions}

Y.L. Liu and S.Z. Zheng performed most of the experimental work. X.Y. Liu analysed the RNA-seq data and wrote the RNA-seq analysis results in the manuscript. X. Wang performed RNA extraction and quantitative PCR experiments. K. Gao helped collect rice materials and provided advice on manuscript organization and writing. W.W. Qi, H.M. Ren and H.R. Hu helped perform salt, mannitol and cold treatment experiments. D.Y. Sun provided advice for the experiments. S.Z. Zheng and J.T. Bai designed all the experiments and wrote the manuscript. All authors have read and approved the manuscript.

\section{Funding}

This work was supported by grants from the National Natural Science Foundation of China (31870243), Natural Science Foundation of Hebei Province (C2018205210 and C2019205055), Start-up funding from Hebei Normal University (L042018B01 and L2019B20) and Postdoctoral Research Foundation of China (2019 M651060).

\section{Availability of data and materials}

The datasets and material used and analyzed in this study are available from the corresponding authors on reasonable request.

Ethics approval and consent to participate

Not applicable.

\section{Consent for publication}

Not applicable.

\section{Competing interests}

The authors declare no competing interests.

\section{Author details}

${ }^{1}$ Ministry of Education Key Laboratory of Molecular and Cellular Biology, Hebei Collaboration Innovation Center for Cell Signaling, Hebei Key Laboratory of Molecular and Cellular Biology, College of Life Sciences, Hebei Normal University, Shijiazhuang 050024, China. ${ }^{2}$ Shijiazhuang No.1 High School, Pingan North Street, Shijiazhuang 050010, China. ${ }^{3}$ South 2nd ring east road 20, Hebei Key Laboratory of Molecular and Cellular Biology, Hebei Normal University, Shijiazhuang 050016, China.

Received: 2 July 2020 Accepted: 19 October 2020

Published online: 11 November 2020

\section{References}

1. Finka A, Goloubinoff P. The CNGCb and CNGCd genes from Physcomitrella patens moss encode for thermosensory calcium channels responding to fluidity changes in the plasma membrane. Cell Stress Chaperones. 2014;19: 83-90.

2. Li B, Gao K, Ren H, Tang W. Molecular mechanisms governing plant responses to high temperatures. J Integr Plant Biol. 2018;60:757-79.

3. Liu HT, Gao F, Cui SJ, Han JL, Sun DY, Zhou RG. Primary evidence for involvement of IP3 in heat-shock signal transduction in Arabidopsis. Cell Res. 2006a;16:394. 
4. Liu HT, Gao F, Li GL, Han JL, Liu DL, Sun DY, et al. The calmodulin-binding protein kinase 3 is part of heat-shock signal transduction in Arabidopsis thaliana. Plant J Cell Mol Biol. 2008;55:760-73.

5. Li B, Gao Z, Liu X, Sun D, Tang W. Transcriptional profiling reveals a time-ofday-specific role of REVEILLE $4 / 8$ in regulating the first wave of heat shockinduced gene expression in Arabidopsis. Plant Cell. 2019;31:2353-69.

6. Kostaki Kl, Coupel-Ledru A, Bonnell VC, Gustavsson M, Sun P, McLaughlin FJ, et al. Guard cells integrate light and temperature signals to control Stomatal aperture. Plant Physiol. 2020;182:1404-19.

7. Tasma IM, Brendel V, Whitham SA, Bhattacharyya MK. Expression and evolution of the phosphoinositide-specific phospholipase C gene family in Arabidopsis thaliana. Plant Physiol Biochem. 2008;46:627-37.

8. Di Fino LM, D'Ambrosio JM, Tejos R, van Wijk R, Lamattina L, Munnik T, et al. Arabidopsis phosphatidylinositol-phospholipase C2 (PLC2) is required for female gametogenesis and embryo development. Planta. 2017;245:717-28.

9. Kanehara K, Yu CY, Cho Y, Cheong WF, Torta F, Shui G, et al. Arabidopsis AtPLC2 is a primary Phosphoinositide-specific phospholipase $\mathrm{C}$ in Phosphoinositide metabolism and the endoplasmic reticulum stress response. PLoS Genet. 2015;11:e1005511.

10. Li L, He Y, Wang Y, Zhao S, Chen X, Ye T, et al. Arabidopsis PLC2 is involved in auxin-modulated reproductive development. Plant J. 2015;84:504-15.

11. Zhang K, Jin C, Wu L, Hou M, Dou S, Pan Y. Expression analysis of a stressrelated phosphoinositide-specific phospholipase $\mathrm{C}$ gene in wheat (Triticum aestivum L.). PloS one. 2014;9:e105061.

12. Zhang Q, van Wijk R, Shahbaz M, Roels W, Schooten BV, Vermeer JEM, et al. Arabidopsis phospholipase $\mathrm{C} 3$ is involved in lateral root initiation and ABA responses in seed germination and Stomatal closure. Plant Cell Physiol. 2018b;59:469-86.

13. Gao K, Liu YL, Li B, Zhou RG, Sun DY, Zheng SZ. Phosphoinositide-specific phospholipase C isoform 3 (AtPLC3) and AtPLC9 function additionally to each other in Thermotolerance in Arabidopsis thaliana. Plant Cell Physiol. 2014:55:1873-83.

14. Zheng SZ, Liu YL, Li B, Shang ZL, Zhou RG, Sun DY. Phosphoinositidespecific phospholipase C9 is involved in the thermotolerance of Arabidopsis. Plant J. 2012;69:689.

15. Singh A, Kanwar P, Pandey A, Tyagi AK, Sopory SK, Kapoor S, et al. Comprehensive genomic analysis and expression profiling of phospholipase $\mathrm{C}$ gene family during abiotic stresses and development in rice. PLoS One. 2013;8:e62494

16. Li L, Wang F, Yan P, Jing W, Zhang C, Kudla J, et al. A phosphoinositidespecific phospholipase $\mathrm{C}$ pathway elicits stress-induced $\mathrm{Ca}(2+)$ signals and confers salt tolerance to rice. New Phytol. 2017;214:1172-87.

17. Deng X, Yuan S, Cao H, Lam SM, Shui G, Hong Y, et al. Phosphatidylinositolhydrolyzing phospholipase C4 modulates rice response to salt and drought. Plant Cell Environ. 2019;42:536-48.

18. Arshad MS, Farooq M, Asch F, Krishna JSV, Prasad PW, Siddique KHM. Thermal stress impacts reproductive development and grain yield in rice. Plant Physiol Biochem. 2017;115:57-72.

19. Liu G, Zha Z, Cai H, Qin D, Jia H, Liu C, et al. Dynamic Transcriptome analysis of anther response to heat stress during Anthesis in Thermotolerant Rice (Oryza sativa L.). Int J Mol Sci. 2020;21:1155.

20. Wahab MMS, Akkareddy S, Shanthi P, Latha P. Identification of differentially expressed genes under heat stress conditions in rice (Oryza sativa L.). Mol Biol Rep. 2020;47:1935-48.

21. Zhou H, Wang X, Huo C, Wang H, An Z, Sun D, et al. A quantitative proteomics study of early heat-regulated proteins by two-dimensional difference gel electrophoresis identified OsUBP21 as a negative regulator of heat stress responses in Rice. Proteomics. 2019;19:e1900153.

22. Feng B, Zhang C, Chen T, Zhang X, Tao L, Fu G. Salicylic acid reverses pollen abortion of rice caused by heat stress. BMC Plant Biol. 2018;18:245.

23. Jagadish KSV, CP, Shi W, Oane R. A phenotypic marker for quantifying heat stress impact during microsporogenesis in rice (Oryza sativa L.). Funct Plant Biol. 2013:41(41):48-55.

24. Zhang C-X, Feng B-H, Chen T-T, Fu W-M, Li H-B, Li G-Y, et al. Heat stressreduced kernel weight in rice at anthesis is associated with impaired source-sink relationship and sugars allocation. Environ Exp Bot. 2018a;155: 718-33.

25. Huo C, Zhang B, Wang H, Wang F, Liu M, Gao Y, et al. Comparative study of early cold-regulated proteins by two dimensional difference gel electrophoresis reveals a key role for phospholipase Da1 in mediating cold acclimation signaling pathway in rice. Mol Cell Proteomics. 2016;15:1397.
26. Zheng Y, Jiao C, Sun H, Rosli HG, Pombo MA, Zhang P, et al. iTAK: a program for genome-wide prediction and classification of plant transcription factors, transcriptional regulators, and protein kinases. Mol Plant. 2016:9:1667-70.

27. Ren H, Gao K, Liu Y, Sun D, Zheng S. The role of AtPLC3 and AtPLC9 in thermotolerance in Arabidopsis. Plant Signal Behav. 2017;12:e1162368.

28. Saidi Y, Finka A, Goloubinoff P. Heat perception and signalling in plants: a tortuous path to thermotolerance. New Phytol. 2011;190:556-65.

29. Liu J, Feng L, Li J, He Z. Genetic and epigenetic control of plant heat responses. Front Plant Sci. 2015;6:267.

30. Liu HT, Guo-Liang LI, Chang H, Sun DY, Zhou RG, Bing LI. Calmodulinbinding protein phosphatase PP7 is involved in thermotolerance in Arabidopsis. Plant Cell Environ. 2007;30:156-64.

31. Liu HT, Huang WD, Pan QH, Weng FH, Zhan JC, Liu Y, et al. Contributions of PIP (2)-specific-phospholipase $C$ and free salicylic acid to heat acclimationinduced thermotolerance in pea leaves. J Plant Physiol. 2006b;163:405-16.

32. Liu HT, Sun DY, Zhou RG. Ca2+ and AtCaM3 are involved in the expression of heat shock protein gene in Arabidopsis. Plant Cell Environ. 2005;28:1276-84.

33. Alexandra J, Lassalles JP, Kado RT. Opening of Ca $2+$ channels in isolated red beet root vacuole membrane by inositol 1,4,5-trisphosphate. Nature. 1990;343:567-70.

34. Hirose K, Kadowaki S, Tanabe M, Takeshima H, lino M. Spatiotemporal dynamics of inositol 1,4,5-trisphosphate that underlies complex Ca2+ mobilization patterns. Science. 1999;284:1527-30.

35. Hirayama T, Ohto C, Mizoguchi T, Shinozaki K. A gene encoding a phosphatidylinositol-specific phospholipase $\mathrm{C}$ is induced by dehydration and salt stress in Arabidopsis thaliana. Proc Natl Acad Sci U S A. 1995;92:3903-7.

36. Xia K, Wang B, Zhang J, Li Y, Yang H, Ren D. Arabidopsis phosphoinositidespecific phospholipase $C 4$ negatively regulates seedling salt tolerance. Plant Cell Environ. 2017:40:1317-31.

37. Banti V, Loreti E, Novi G, Santaniello A, Alpi A, Perata P. Heat acclimation and cross-tolerance against anoxia in Arabidopsis. Plant Cell Environ. 2008; 31:1029-37.

38. Ran X, Zhao F, Wang Y, Liu J, Zhuang Y, Ye L, et al. Plant Regulomics: a data-driven interface for retrieving upstream regulators from plant multiomics data. Plant J. 2020;101:237-48.

39. Liu Z, Zhao J, Li Y, Zhang W, Jian G, Peng Y, et al. Non-uniform distribution pattern for differentially expressed genes of transgenic Rice Huahui 1 at different developmental stages and environments. PLoS One. 2012;7:e37078.

40. Asano T, Hakata M, Nakamura H, Aoki N, Komatsu S, Ichikawa H, et al. Functional characterisation of OsCPK21, a calcium-dependent protein kinase that confers salt tolerance in rice. Plant Mol Biol. 2011;75:179-91.

41. Arnon DI. Copper enzymes in isolated chloroplasts. Polyphenoloxidase in Beta vulgaris. Plant Physiol. 1949;24:1-15.

42. Robinson MD, McCarthy DJ, Smyth GK. edgeR: a Bioconductor package for differential expression analysis of digital gene expression data. Bioinformatics. 2010;26(1):139-40.

43. Tian T, Liu Y, Yan H, You Q, Yi X, Du Z, et al. agriGO v2.0: a GO analysis toolkit for the agricultural community, 2017 update. Nucleic Acids Res. 2017; 45:W122-9.

\section{Publisher's Note}

Springer Nature remains neutral with regard to jurisdictional claims in published maps and institutional affiliations.

Ready to submit your research? Choose BMC and benefit from:

- fast, convenient online submission

- thorough peer review by experienced researchers in your field

- rapid publication on acceptance

- support for research data, including large and complex data types

- gold Open Access which fosters wider collaboration and increased citations

- maximum visibility for your research: over $100 \mathrm{M}$ website views per year

At BMC, research is always in progress.

Learn more biomedcentral.com/submissions 